\title{
Validación del instrumento de medición para la caracterización nacional de estudiantes de derecho
}

\author{
Validation of the instrument of measurement to a national \\ characterization of law students
}

María Francisca Elgueta Rosas ${ }^{1}$ y Francisco Zamorano Figueroa ${ }^{2}$

Resumen: el presente artículo representa el recorrido metodólogico realizado por los autores para la construcción del cuestionario utilizado en la primera Encuesta Nacional de Caracterización de Estudiantes de Derecho en Chile. A lo largo del texto, se consideran los procedimientos que permitieron entregar validez y fiabilidad al instrumento de medición. Finalmente, se exponen análisis estadísticos que miden la consistencia interna de las escalas destinadas a medir conceptos más complejos dentro del cuestionario.

Palabras claves: Encuesta Nacional de Caracterización de Estudiantes de Derecho, validez, fiabilidad.

Abstract: this paper shows the methodological path of authors in order to create the questionnaire used in the first National Survey of Characterization of Law Students in Chile. In the text, it implies procedures which allowed giving validity and reliability to the measurement instrument. Finally, statistical analyses are shown in order to measure the internal consistency of scales devoted to measure complex concept within questionnaire.

Key words: National Survey of Characterization of Law Students, validity, reliability.

\footnotetext{
${ }^{1}$ Doctora en Educación, Universidad de Valladolid; Diplomada en Docencia Universitaria, Universidad Metropolitana de Ciencias de la Educación; Diplomada en Gestión Universitaria, Universidad Metropolitana de Ciencias de la Educación; Diplomada en Diseño e Implementación de Encuestas, Pontificia Universidad Católica de Chile. Investigadora asociada a la Unidad de Pedagogía Universitaria y Didáctica del Derecho, Facultad de Derecho, Universidad de Chile.

${ }^{2}$ Magíster en Metodología de la Investigación Social, Lodon School of Economics and Political Science (LSE), Sociólogo Universidad de Chile, Investigador asociado a Unidad de Pedagogía Universitaria y Didáctica del Derecho, Facultad de Derecho, Universidad de Chile.
} 


\section{Introducción}

La Facultad de Derecho de la Universidad de Chile ha institucionalizado su interés en el desarrollo de la investigación en pedagogía universitaria y didáctica del Derecho por medio de la creación de una Unidad especializada en esta temática. Precisamente, la Unidad de Pedagogía Universitaria y Didáctica del Derecho (UPUDD) de la Facultad de Derecho ha sido la primera institución a nivel nacional en implementar, como iniciativa propia, una investigación con objeto de caracterizar a los estudiantes de Derecho que asisten a las universidades chilenas.

Esta iniciativa se transformó en una necesidad al constatar que la ausencia de investigación sistemática sobre los estudiantes de Derecho es una constante no sólo a nivel general, sino que en un nivel particular dentro de las mismas escuelas de Derecho. Esto significó una motivación para la UPUDD en la medida que la información a generar podría representar un aporte fundamental para el medio académico de la enseñanza del Derecho.

En efecto, no se puede conceptualizar la docencia sin la presencia y la participación del estudiantado en el proceso formativo. De este modo, resulta imperativo contribuir con datos estadísticos que permitan profundizar en el conocimiento de nuestros estudiantes con el objetivo último de alcanzar altos estándares en la práctica de la docencia jurídica.

Esto se suma a la pretensión de aportar a una reflexión conjunta con las distintas Facultades de Derecho, puesto que las autoridades de cada institución deben hacerse cargo y aceptar la diversidad cultural y heterogeneidad social que caracteriza al alumnado de Derecho a lo largo del país.

Por otra parte, surgen razones profesionales para llevar a cabo una investigación como ésta en la medida que el estudio se erige como un insumo necesario para la Corte Suprema, el Poder Judicial, el Congreso Nacional, etc. Esto supone que nuestras instituciones entreguen un valor adecuado a la formación profesional del estudiante de Derecho en tanto son ellos mismos quienes ocuparán las posiciones de trabajo dentro de estos organismos.

También, este estudio contribuye a una mejor comprensión de la presencia de la cultura jurídica en la formación universitaria. Al respecto, nuestros estudiantes son un componente importante para la formación de una cultura jurídica entendida como un sistema independiente, por lo que sus acciones, percepciones y sistemas valorativos aparecen como aspectos importantes a estudiar en la investigación.

En lo que respecta al plano netamente universitario, la primera Encuesta Nacional de Caracterización de Estudiantes de Derecho (ENCED) se propuso como objetivo dar 
cuenta de las fortalezas y debilidades percibidas por el estudiante de Derecho en relación al sistema universitario, de modo que fuese posible generar información y definiciones en torno al perfil de ingreso del estudiante, al seguimiento de su experiencia universitaria y al egreso en la formación profesional.

Además, la ENCED entrega datos que colaboran con los procesos de gestión de la docencia en las distintas instituciones jurídicas formadoras sin importar su carácter técnico o profesional.

Por último, los aportes de este estudio se conectan con posibilitar la reflexión alrededor del aseguramiento de la calidad de las decisiones en los procesos de enseñanzaaprendizaje en la formación jurídica.

En lo concreto, la primera Encuesta Nacional de Caracterización de Estudiantes de Derecho tiene como objetivo principal llevar a cabo una descripción exhaustiva del conjunto de estudiantes de las escuelas de derecho de las universidades chilenas que se encuentren cursando segundo año o algún nivel superior de la carrera. Esta caracterización contempla diversas temáticas de estudio que pueden ser resumidas en torno a seis áreas: i) área demográfica y socioeconómica; ii) área de evaluación institucional y procesos de gestión de la carrera; iii) área del estudiante en sus habilidades y estrategias de aprendizaje; iv) área de la percepción de la docencia y vida académica; v) área de la autoidentificación y autoclasificación de los estudiantes; y vi) área de la participación social y política de los estudiantes

La caracterización de los universitarios se desarrolló a partir del diseño de una encuesta particular y de la aplicación de un total de 2141 cuestionarios a los estudiantes de derecho de las universidades nacionales.

A partir de la experiencia del diseño en encuesta y la información recolectada, el presente artículo tiene como objetivo detallar el proceso de construcción y validación del cuestionario utilizado para caracterizar a los estudiantes chilenos de Derecho.

\section{Metodología}

La primera Encuesta Nacional de Caracterización de Estudiantes de Derecho es un estudio no experimental y transversal llevado a cabo por la Unidad de Pedagogía Universitaria y Didáctica del Derecho de la Facultad de Derecho de la Universidad de Chile. El carácter no experimental de la investigación es definido por la nula pretensión de "control directo de las variables independientes debido a que sus manifestaciones ya han 
ocurrido o a que son inherentemente no manipulables"”. Además, si bien es una encuesta que tiene pretensiones de repetirse a lo largo del tiempo, su diseño es transversal ya que es la primera de su tipo, por lo que se hace "un corte perpendicular de una situación en un momento dado y se estudia su estructura"4. Finalmente, cabe consignar que, como su nombre lo indica, el universo del estudio está compuesto por los estudiantes de derecho de las escuelas de derecho a lo largo del país.

Sin embargo, es necesario aclarar que la población objetiva se reduce a aquellos estudiantes de las 92 escuelas diurnas de derecho, que se encuentran cursando desde segundo año de la carrera en adelante durante el año 2012. Esta delimitación implica reducir el número de estudiantes matriculados en la modalidad diurna, puesto que los alumnos de primer año no fueron considerados para efectos de estos estudios. En este sentido, se trabajó con una población finita que asciende a 23.253 estudiantes de los 29.618 del total que componen la jornada diurna.

A su vez se consideró una segunda restricción, puesto que se consideraron sólo las escuelas diurnas de derecho, cuya matrícula fuera superior o igual al 0,5\% de la matricula total. Esta nueva delimitación conlleva una nueva reducción de los sujetos incluidos en el estudio, puesto que 60 escuelas cumplen con el criterio propuesto. Esto significa que el marco de muestreo a estudiar alcanzó un total de 21.817 estudiantes de derecho para el año 2012.

Para el proceso de selección de la muestra se determinó realizar un muestreo estratificado por conglomerados bietápico. Mediante la elección de un procedimiento estratificado se busca "atenuar la variabilidad dentro de una población", , de modo que en ENCED no aparezcan escuelas sobre o infra representadas. El apellido bietápico obedece a que el muestreo se dividió en dos etapas (probabilístico estratificado en una primera etapa y no probabilístico por cuotas en una segunda), donde las escuelas de derecho constituyen las Unidades de Muestreo Primaria (UMP) y los estudiantes son las Unidades de Muestreo Secundaria (UMS).

Con respecto a la definición de estratos para la primera etapa del muestreo, se establecieron cuatro estratos definidos por la combinación de dos variables dicotómicas: 1) la variable clasificación de la institución, que distingue entre Universidades CRUCH y universidades privadas y 2) la variable ubicación de la sede, que distingue entre

\footnotetext{
${ }^{3}$ ALBERT GÓMEZ, María José. La Investigación Educativa: Claves Teóricas. Madrid: Mc Graw Hill, 2007. P. 91

${ }^{4}$ SIERRA, Restituto. Técnicas de investigación social: teoría y ejercicios. Madrid: Thomson Editores, 2007. P. 34

${ }^{5}$ PARDINAS, Felipe. Metodología y técnicas de investigación en ciencias sociales. México: Siglo Veintiuno Editores, 2008. P. 182.
} 
universidades pertenecientes a la Región Metropolitana y otras pertenecientes a alguna otra región.

De este modo, en una primera fase, se seleccionó probabilísticamente las UMP mediante Muestreo Aleatorio Simple (MAS) de acuerdo a la cantidad de escuelas que componen cada estrato en el marco de muestreo (59 escuelas). Además, cabe consignar que se procedió con un mecanismo de reemplazo en caso de rechazo o imposibilidad de contacto. Al término de esta etapa, la muestra se constituyó de 31 escuelas de Derecho pertenecientes a las 4 posibles combinaciones definidas anteriormente (estratos), a saber: escuela CRUCH de región Metropolitana, escuela CRUCH de otra región, escuela privada de región Metropolitana y escuela privada de otra región.

Una vez definidas las UMP, se desarrolló una segunda etapa no probabilística de selección de UMS (estudiantes) por medio de un muestreo por cuotas.

El muestreo por cuotas es un muestreo estratificado en que la elección de las unidades se encomienda al entrevistador, y en el que la entidad de la cuota impide una libertad absoluta de elección y hace que la muestra total, limitándonos a las variables planteadas al establecer las cuotas, reproduzca la distribución de la población. ${ }^{6}$

Así, se obtuvo una cuota para cada uno de los estratos de estudiantes que debían considerarse en el estudio. Sumando cada cuota por estrato, se calculó un tamaño de la muestra total, correspondiendo a 2141 estudiantes.

\section{Procedimientos de validación del instrumento de medición}

Como se mencionó con anterioridad, la primera ENCED supone seis áreas de caracterización que determinan los diversos aspectos a medir en la investigación. Esto conlleva una enorme tarea en términos de la validez del estudio y la cantidad de variables que forman parte del cuestionario, puesto que el concepto de validez "hace referencia al grado en el que un determinado procedimiento de traducción de un concepto en variable, registra efectivamente el concepto que se pretende registrar"7. Es decir, la validación del instrumento de medición se complejiza debido a las múltiples aristas cubiertas por la ENCED.

\footnotetext{
${ }^{6}$ CORBETTA, Piergiorgio. Metodología y técnicas de investigación social. Madrid: Mc Graw Hill, 2003. P. 306.

${ }^{7}$ Ibíd. P. 107.
} 
Generalmente el error de validez se sitúa en el paso del concepto al indicador y nace del 'error de indicación' (...) La validez de un determinado indicador es, en efecto, mucho más complicado de reconocer, y es muy difícil de medir. ${ }^{8}$

Para tales efectos, en un primer momento se realizó una búsqueda exhaustiva de literatura que permitió entender de mejor forma los conceptos a estudiar. El análisis teórico resulta fundamental para el sustento de la validez del estudio en la medida que justifica "por qué esas preguntas pueden informar adecuadamente del concepto a medir".

El procedimiento de análisis teórico se complementó con una revisión bibliográfica de diferentes artículos sobre construcción de instrumentos afines a los diferentes objetos de estudio. Esto permitió que los diversos conceptos definidos en la primera etapa se correspondieran con antecedentes empíricos que permiten mejorar la medición de ellos.

De este modo, en la primera etapa de revisión de literatura se llevó a cabo la definición conceptual de las diversas variables incluidas en la investigación. Las variables se ajustaron a los diversos elementos que forman parte de las áreas ya mencionadas. Luego, un segundo momento consistió en el desarrollo del proceso de definición operacional de estas variables, de manera que se identificaron indicadores y/o factores pertinentes para su medición. Para el caso de conceptos complejos y que no poseen propiedades manifiestas observables se adaptaron escalas de medición validadas por diferentes metodólogos. La adaptación de preguntas y escalas es un procedimiento recurrente en la investigación cuantitativa pues permite:

concentrar los esfuerzos creativos en sólo la parte de la tarea y además brindará la posibilidad de comparar nuestros resultados con los de otras investigaciones, lo que casi siempre es muy útil a la hora de interpretar y valorar lo obtenido. ${ }^{10}$

Dicho procedimiento aumenta la probabilidad de mejorar la validez en las mediciones a realizar.

Entre las fuentes consultadas para la inclusión de preguntas en el cuestionario de caracterización se cuentan la Encuesta Nacional de Juventud, desarrollada por el Instituto Nacional de Juventud ${ }^{11}$ y la encuesta CEFECH construida por el Centro de Estudios de la Federación de Estudiantes de Chile ${ }^{12}$. Con respecto a las escalas utilizadas, se consultó

\footnotetext{
${ }^{8}$ Ibíd. P. 108.

9 ASÚN INOSTROZA, Rodrigo. "Construcción de cuestionarios y escalas: el proceso de la producción de información cuantitativa”. En: Metodologías de Investigación Social: Introducción a Los Oficios. Santiago: LOM Ediciones, 2014. P. 112

${ }^{10}$ Ibíd. P. 108.

${ }^{11}$ INJUV, GOBIERNO DE CHILE; Sexta Encuesta Nacional de Juventud. Disponible en web http://www.injuv.gob.cl/modules.php [consultado en diciembre de 2014]

${ }^{12}$ Para mayor información, ver: CENTRO DE ESTUDIOS DE LA FEDERACIÓN DE ESTUDIANTES DE LA UNIVERSIDAD DE CHILE. Encuesta de Caracterización de Estudiantes Universidad de Chile 2011:
} 
tanto las escalas originales como aquellas adaptaciones realizadas por metodólogos para las características de la población que se propone estudiar.

Una vez diseñado el cuestionario, se procedió a una evaluación de las preguntas incluidas por parte de un experto metodólogo, quien entregó críticas, recomendaciones y sugerencias con respecto al instrumento y a un potencial análisis de los datos posteriores. La consulta a expertos tiene como objeto mejorar la validez del instrumento ya que son "quienes respaldan, a partir de sus conocimientos previos, que el instrumento es adecuado para medir lo que se desea medir",

Finalmente, se probó el cuestionario por medio de una prueba piloto o pretest. La prueba piloto es un procedimiento metodológico que involucra aplicar el instrumento a sujetos que comparten características con la población a estudiar. Con ello se busca someter

a prueba no sólo el instrumento de medición, sino también las condiciones de aplicación y los procedimientos involucrados. Se analiza si las instrucciones se comprenden y si los ítems funcionan de manera adecuada, se evalúa el lenguaje y la redacción. ${ }^{14}$

En la ENCED, la prueba piloto se llevó a cabo mediante la aplicación del cuestionario a una de las escuelas de derecho. Dentro de esta universidad se encuestaron a 112 alumnos y alumnas, que corresponden a la totalidad de estudiantes de derecho en segundo año o curso superior de la institución. Es decir, se realizó un censo de la población objetiva en dicha escuela de derecho.

De esta manera, fue posible generar un instrumento de medición propio que consta de 58 preguntas, destinadas a medir seis áreas de investigación, primando la idea de que "un test es válido si mide lo que pretende medir" 15 .

\section{Procedimientos de validación y fiabilidad de escalas}

De la totalidad de variables incluidas en la ENCED, se consideró un grupo de variables definidas para medir conceptos complejos a través de escalas de medición. La particularidad de la medición por escalas es que permite realizar estimaciones de su validez y fiabilidad por medio de procedimientos estadísticos.

Análisis y Principales Resultados, 2012. Disponible en web: http://fech.cl/wp-

content/uploads/2012/03/INFORME-FINAL-ENCUESTA-CEFECH.pdf [consultado en diciembre de 2014]

${ }^{13}$ ASÚN, R. Ibíd. P. 112.

${ }^{14}$ HERNÁNDEZ SAMPIERI, Roberto; FERNÁNDEZ COLLADO, Carlos y BAPTISTA, Lucio Pilar.

Metodología de la Investigación. México: Ed. McGraw Hill, 2006. P. 306.

15 BEST, John W. Cómo Investigar en Educación. Madrid: Ediciones Morata, 1992. P.167 
La noción de fiabilidad "de un instrumento de medición se refiere al grado en que su aplicación repetida al mismo sujeto u objeto produce resultados iguales"16. Se busca entonces determinar la "la consistencia o estabilidad de una medida del comportamiento"17. En este sentido, este apartado pretende generar estimaciones numéricas de la precisión del instrumento de medición a partir de los resultados obtenido en la ENCED.

$\mathrm{Al}$ respecto, es necesario señalar que

generalmente, se asocia la fiabilidad al error 'aleatorio' y la validez al error 'sistemático'. Por ello, la fiabilidad es más fácilmente verificable que la validez, puesto que el error aleatorio puede localizarse a través de repeticiones de la observación sobre el mismo sujeto (las variaciones entre las repeticiones se deben al error aleatorio); mientras que la validez es más difícil de identificar, puesto que el error sistemático, que está a su base, se presenta constantemente en cada observación, y por tanto hace que no se pueda conocer el estado efectivo de la propiedad estudiada. ${ }^{18}$

Por esta razón, en la mayoría de las escalas a analizar, se aplicará primordialmente un análisis de fiabilidad antes que uno de validez, así como la literatura utilizada para comparar resultados se centrará principalmente en la primera antes que en la segunda.

Las escalas a evaluar son cuatro: a) Escala de Estrategias de Adquisición de Información; b) Escala de Autoritarismo de Derechas; c) Escala de LiberalismoConservadurismo y d) Escala de Percepción de Atributos de la Sociedad Chilena.

\section{a) Escala de Estrategias de Adquisición de Información}

La Escala de Estrategias de Adquisición de Información es una sección de la escala de estrategias de aprendizajes ACRA (Adquisición, Codificación, Recuperación y Apoyo) ${ }^{19}$ orientada a medir cómo los estudiantes integran la información estudiada. Las estrategias de adquisición de información se componen de dos tipos de estrategias, denominadas estrategias atencionales y estrategias de repetición.

Las estrategias atencionales contemplan la incorporación de la información por medio del dominio de la totalidad del aparato cognitivo, de modo que los procesos atencionales se enfoquen sobre lo que se está estudiando. Se distinguen dos procedimientos de acercamiento a la información: la exploración y la fragmentación.

\footnotetext{
${ }^{16}$ Ibíd. P. 277.

${ }^{17}$ COZBY, Paul C. Métodos de Investigación del Comportamiento. México: Ed. McGraw Hill, 2004. P. 95.

${ }^{18}$ CORBETTA. Ibíd. P. 107.

${ }^{19}$ Para mayor información, ver: J ROMÁN, José María y GALLEGO, Sagrario. Escala de estrategias de aprendizaje. Madrid: TEA Ediciones, 1994
} 
Por otro lado, las estrategias de repetición consisten en procedimientos de reiteración del conocimiento a aprender. Entre las tácticas utilizadas se pueden contar el repaso en voz alta, el repaso mental y el repaso reiterado.

Precisamente, cada estrategia posee una cantidad contable de tácticas que se presentan como factores de aprendizaje. En este sentido, la escala de estrategias de adquisición de información consta de siete factores que compendian la información de veinte ítemes destinados a medir la escala.

Antes de adentrarse en el análisis de la validez de la escala, es necesario aclarar que la escala ACRA es una escala de medición creada originalmente para observar estrategias de aprendizaje de estudiantes secundarios en España, por lo que se han desarrollado múltiples adaptaciones que involucran estudiantes universitarios y de otras regiones del mundo.

En primer lugar, cabe mencionar que los creadores de la escala ACRA probaron la validez de constructo, contenido y predicción mediante la aplicación del instrumento a una muestra de 650 estudiantes. Esfuerzos similares han sido desarrollados por otros investigadores que han intentado probar la validez del instrumento a muestras de estudiantes universitarios e, incluso, validando versiones abreviadas de la escala ACRA ${ }^{20}$.

En lo que respecta a la fiabilidad de la escala de estrategias de adquisición, se realizó un análisis de fiabilidad mediante un análisis de consistencia interna (a de Cronbach). Así, el índice de fiabilidad para la escala muestra un nivel aceptable en la tabla 1 con un alfa global $=0.825$ (muy similar a otros estudios).

Tabla 1: Estadísticos de fiabilidad de la escala de estrategias de adquisición de la información.

\begin{tabular}{|r|r|}
\hline $\begin{array}{c}\text { Alfa de } \\
\text { Cronbach }\end{array}$ & $\begin{array}{c}\text { N de } \\
\text { elementos }\end{array}$ \\
\hline, 825 & 20 \\
\hline
\end{tabular}

Mediante la tabla 2, se exponen los estadísticos descriptivos de las variables que conforman la escala de estrategias de adquisición de información, así como la correlación de los ítemes medidos en el cuestionario. La media y la desviación típica nos ayudan a entender la distribución de los datos dentro de cada una de las variables incluidas en el análisis. El estadístico correlación ítem-total indica el nivel de asociación que cada uno de los ítemes posee con respecto al puntaje total de la escala. Finalmente, a de Cronbach sin el ítem indica el valor que asumiría el alfa de Cronbach global si se eliminará el ítem del análisis. Esta situación resulta clave en la medida que la eliminación de uno o dos ítemes

\footnotetext{
${ }^{20}$ Para mayor información, ver: CLOSAS, Antonio Humberto et al. "Validación de un cuestionario sobre estrategias de aprendizaje para estudiantes universitarios”. En: SOSA, Carola y DALFARO, Nidia (comps.). La Universidad Tecnológica Nacional - U.T.N. - En El NorEste Argentino - N.E.A. Disponible en web: http://www.edutecne.utn.edu.ar/investigacion_fr_res/Closas_Ciencias_Sociales.pdf [consultado en diciembre de 2014]
} 
podría aumentar el número del alfa global, es decir, aumentaría la estabilidad de la medición.

Al respecto, cabe señalar que gran parte de los ítemes muestran una correlación al menos moderada con el total, con la excepción de los ítemes Adq1 (0.24), Adq3 (0.19) y Adq4 (0.29). Si bien esto supone un resultado no esperado, puesto que afecta principalmente al factor exploración que contiene los ítemes 1 y 3, la situación es aceptable para los restantes 17 ítemes, en tanto reflejan correlaciones superiores al 0.33 con el puntaje total. Esta situación se hace evidente entre los ítemes que componen el factor repaso en voz alta, donde todos sus elementos correlacionan sobre el 0.45 con el total de la escala.

Tabla 2: Media, desviación típica, correlación ítem-total y a de Cronbach sin el ítem para cada ítem de la escala de estrategias de adquisición de información.

\begin{tabular}{|c|c|c|c|c|c|}
\hline Factor & Ítem & Media & $\begin{array}{c}\text { Desviación } \\
\text { típica }\end{array}$ & $\begin{array}{c}\text { Correlación } \\
\text { ítem-total }\end{array}$ & $\begin{array}{c}\text { a de } \\
\text { Cronbach } \\
\text { sin el ítem }\end{array}$ \\
\hline \multirow{3}{*}{ Exploración } & Adq1 & 2,493 & 1,015 & 0,242 & 0,826 \\
\hline & Adq3 & 2,744 & 1,103 & 0,193 & 0,829 \\
\hline & Adq11 & 3,254 & 0,865 & 0,485 & 0,814 \\
\hline \multirow{2}{*}{$\begin{array}{c}\text { Subrayado } \\
\text { líneal }\end{array}$} & Adq5 & 3,542 & 0,797 & 0,394 & 0,818 \\
\hline & Adq8 & 3,510 & 0,818 & 0,393 & 0,818 \\
\hline \multirow{3}{*}{$\begin{array}{c}\text { Subrayado } \\
\text { Idiosincrático }\end{array}$} & Adq6 & 3,269 & 0,973 & 0,430 & 0,816 \\
\hline & Adq7 & 3,175 & 1,109 & 0,334 & 0,821 \\
\hline & Adq10 & 2,680 & 1,070 & 0,391 & 0,818 \\
\hline \multirow{2}{*}{ Epigrafiado } & Adq2 & 2,655 & 1,044 & 0,425 & 0,816 \\
\hline & Adq9 & 2,562 & 1,069 & 0,484 & 0,813 \\
\hline \multirow{4}{*}{$\mid \begin{array}{c}\text { Repaso en voz } \\
\text { alta }\end{array}$} & Adq13 & 2,909 & 1,063 & 0,458 & 0,814 \\
\hline & Adq14 & 2,913 & 1,016 & 0,503 & 0,812 \\
\hline & Adq16 & 2,762 & 0,982 & 0,476 & 0,814 \\
\hline & Adq19 & 2,275 & 1,041 & 0,459 & 0,814 \\
\hline \multirow{4}{*}{$\begin{array}{l}\text { Repaso } \\
\text { mental }\end{array}$} & Adq4 & 2,919 & 0,999 & 0,294 & 0,823 \\
\hline & Adq15 & 3,059 & 0,904 & 0,416 & 0,817 \\
\hline & Adq17 & 2,433 & 0,915 & 0,349 & 0,820 \\
\hline & Adq18 & 2,489 & 0,972 & 0,442 & 0,815 \\
\hline \multirow{2}{*}{$\begin{array}{l}\text { Repaso } \\
\text { reiterado }\end{array}$} & Adq12 & 3,463 & 0,735 & 0,439 & 0,817 \\
\hline & Adq20 & 3,041 & 0,909 & 0,396 & 0,818 \\
\hline
\end{tabular}

Por otra parte, al momento de observar en qué medida variarían los resultados si se eliminará alguno de los ítemes que presentan bajas correlaciones, se advierte que el aumento del valor del a de Cronbach no es sustantivo, puesto que apenas se incrementaría 
desde 0.825 a 0.829 si se excluye del análisis al ítem con menos correlación ítem-total (Adq3). Esto se explica porque el estadístico a de Cronbach ya es bastante alto al considerar los veinte ítemes que lo componen (mayor a 0.70), por lo que la fiabilidad de la escala es aceptable aun cuando no se eliminen los ítemes con baja correlación con la puntuación global.

\section{b) Escala de Autoritarismo de Derechas}

La Escala de Autoritarismo de Derechas (RWA) se desarrolló con el objetivo de medir el autoritarismo de derechas desde un punto de vista psicológico y social. La escala se divide en tres subdimensiones: la sumisión a la autoridad, el convencionalismo con respecto al orden establecido y las actitudes agresivas que intentan preservarlo. Bajo esta definición de autoritarismo, cabe la posibilidad de que personas que se definen de izquierda presenten niveles elevados de autoritarismo. En este sentido, la escala se relaciona con la medición de personalidades e ideologías autoritarias ${ }^{21}$.

Si bien es cierto la escala original se compone de treinta ítemes, en el cuestionario de la ENCED se utilizó una versión abreviada y adaptada con el objeto de apartar sesgos idiosincráticos presentes en la primera versión. Mediante esta elección se pretende mejorar la validez, en tanto se excluyen elementos ambiguos que podrían ser influenciados por determinantes culturales.

De este modo, la versión utilizada fue diseñada por Manuel Cárdenas y Luis Parra, para un contexto de jóvenes estudiantes en Chile $(n=264)^{22}$. En esta escala se consideran 12 ítemes (donde $1=$ muy de acuerdo y $7=$ muy en desacuerdo) divididos en tres factores que explicaron el $50.75 \%$ de la varianza total en un primer análisis factorial. Los tres factores se corresponden con una agrupación de ítemes que permite identificar las tres subdimensiones teóricas señaladas anteriormente: sumisión, convencionalismo y agresión. Luego, a través de un análisis factorial confirmatorio se testeó el modelo de tres factores, concluyendo que el ajuste es mejor en este modelo. Se asegura así trabajar con una escala validada para una población similar a la de la ENCED.

Con respecto a la fiabilidad de la escala, la versión abreviada utilizada mostró una fiabilidad de 0.72 (a de Cronbach). Estos números son similares a los obtenidos en la ENCED, en la media se obtuvo un a de Cronbach $=0.77$. Con ello se confirma la consistencia interna de la escala.

\footnotetext{
${ }^{21}$ Para mayor información, ver: ALTEMEYER, Bob. Right-Wing Authoritarianism. Winnipeg: University of Manitoba Press, 1981.

${ }^{22}$ Para mayor información, ver: CÁRDENAS, Manuel y PARRA, Luis; “Adaptación y validación de la versión abreviada de la escala de autoritarismos de derechas (RWA) en una muestra chilena". En: Revista de Psicología, Universidad de Chile. 2010, vol. 19, núm. 1. Pp. 61-79.
} 
Tabla 3: Estadísticos de fiabilidad de la escala RWA

\begin{tabular}{|r|r|}
\hline $\begin{array}{c}\text { Alfa de } \\
\text { Cronbach }\end{array}$ & $\begin{array}{c}\text { N de } \\
\text { elementos }\end{array}$ \\
\hline, 770 & 12 \\
\hline
\end{tabular}

La tabla cuatro muestra la correlación ítem-total de cada uno de los doce ítemes con respecto al puntaje global de la escala RWA. Así, se observa que diez de los doce elementos reflejan correlaciones superiores al 0.36, mientras doce ítemes de la dimensión sumisión presentan correlaciones bajas (inferiores a 0.27). Cabe destacar que el ítem AD12 presentó una correlación fuerte en relación al total $(\mathrm{r}=0.567)$.

En lo que refiere a la variación del a de Cronbach si se elimina algún ítem, se evidenció que la fiabilidad mejoraría al eliminar un elemento (AD5). Sin embrago, nuevamente se decidió incluir este ítem, puesto que la mejora en el nivel de fiabilidad no es sustantiva (0.007). Además, el a de Cronbach calculado alcanza un nivel aceptable al superar el 0.70 de consistencia interna. De este modo, para el análisis final de los resultados fueron considerados los doce ítemes ya validados por Cárdenas y Parra.

Tabla 4: Media, desviación típica, correlación ítem-total y a de Cronbach sin el ítem para cada ítem de la escala RWA.

\begin{tabular}{|c|c|c|c|c|c|}
\hline Dimensión & Ítem & Media & $\begin{array}{c}\text { Desviación } \\
\text { típica }\end{array}$ & $\begin{array}{c}\text { Correlación } \\
\text { ítem-total }\end{array}$ & $\begin{array}{c}\text { a de } \\
\text { Cronbach } \\
\text { sin el ítem }\end{array}$ \\
\hline \hline \multirow{3}{*}{ Agresión } & AD1 & 3,967 & 2,116 & 0,395 & 0,755 \\
& AD2 & 5,145 & 1,979 & 0,429 & 0,751 \\
& AD4 & 5,349 & 2,035 & 0,472 & 0,747 \\
& AD6 & 4,984 & 1,934 & 0,452 & 0,749 \\
& AD11 & 4,132 & 1,867 & 0,368 & 0,758 \\
\hline \multirow{3}{*}{ Sumisión } & AD3 & 3,856 & 2,121 & 0,265 & 0,770 \\
& AD5 & 3,676 & 2,192 & 0,200 & 0,777 \\
& AD9 & 3,997 & 2,111 & 0,380 & 0,757 \\
& AD12 & 4,549 & 2,127 & 0,567 & 0,735 \\
\hline \multirow{3}{*}{ Convencionalismo } & AD7 & 4,942 & 1,980 & 0,389 & 0,756 \\
& AD8 & 4,384 & 1,968 & 0,530 & 0,741 \\
& AD10 & 5,333 & 2,036 & 0,460 & 0,748 \\
\hline
\end{tabular}




\section{c) Escala de Liberalismo-Conservadurismo}

A continuación se presentas dos escalas construidas a partir de variables provenientes de la encuesta nacional de juventud y de la encuesta CEFECH. La particularidad de estas escalas es que aportan antecedentes que permitan comparar procedimientos de validez y fiabilidad, por lo que en estos apartados se tratará de contribuir a la discusión metodológica mediante el análisis de fiabilidad por medio del a de Cronbach.

Así, se trabajó con una Escala de Liberalismo-Conservadurismo, compuesta por cinco variables que medían el nivel de acuerdo frente a algunas posibilidades de legislar con respecto a temas valóricos de interés general (donde 1=muy de acuerdo; 4=muy en desacuerdo). Las variables incluidas en el análisis fueron el grado de acuerdo con: ley de divorcio, legalización del matrimonio homosexual, derecho a adopción homosexual, legalización de eutanasia y aborto por decisión voluntaria.

La escala mostró altos niveles de fiabilidad en la medida que se observó un a de Cronbach $=0.810$. Además, la tabla seis muestra que la correlación ítem-total es alta en los 5 elementos considerados $(r>0.5)$. Inclusive, la eliminación de alguno de los ítemes no significaría un aumento de la consistencia interna de la escala.

Tabla 5: Estadísticos de fiabilidad de la escala de Liberalismo-Conservadurismo.

\begin{tabular}{|r|r|}
\hline Alfa de Cronbach & $\begin{array}{c}\mathrm{N} \text { de } \\
\text { elementos }\end{array}$ \\
\hline, 810 & 5 \\
\hline
\end{tabular}

Tabla 6: Media, desviación típica, correlación ítem-total y a de Cronbach sin el ítem para cada ítem de la Escala de Liberalismo-Conservadurismo.

\begin{tabular}{|l|c|c|c|c|}
\hline \multicolumn{1}{|c|}{ Ítem } & Media & $\begin{array}{c}\text { Desviación } \\
\text { típica }\end{array}$ & $\begin{array}{c}\text { Correlación } \\
\text { ítem-total }\end{array}$ & $\begin{array}{c}\text { a de } \\
\text { Cronbach } \\
\text { sin el ítem }\end{array}$ \\
\hline \hline $\begin{array}{l}\text { Ley de divorcio } \\
\text { Legalización del } \\
\text { matrimonio de personas } \\
\text { del mismo sexo } \\
\text { Derecho a la adopción } \\
\text { de hijos por parte de } \\
\text { padres del mismo sexo }\end{array}$ & 1,388 & 0,713 & 0,534 & 0,800 \\
$\begin{array}{l}\text { Legalización de la } \\
\text { eutanasia } \\
\text { Aborto por decisión }\end{array}$ & 2,426 & 1,185 & 0,723 & 0,731 \\
\begin{tabular}{l} 
voluntaria de la pareja \\
\hline
\end{tabular} & 2,708 & 1,216 & 0,663 & 0,752 \\
\hline
\end{tabular}




\section{d) Escala de Percepción de Atributos de la Sociedad Chilena}

Finalmente, el análisis se repite para la escala de Percepción de Atributos de la Sociedad Chilena. Esta escala se compone de diez ítemes destinados a medir la lejanía o cercanía con la que los entrevistados evalúan atributos del país (donde 1=muy cercano; 10=muy lejano). Los elementos considerados para el análisis refieren a los atributos: democrático, austero, igualitario en oportunidades, libre, tolerante, no clasista, solidario, justo, seguro y moderno.

La fiabilidad para esta escala es consistente en la medida que supera el 0.70 (a de Cronbach=0.881). Por otra parte, la tabla 8 muestra que la correlación ítem-total es alta en los diez elementos considerados ( $r>0.5)$. Nuevamente, la eliminación de alguno de los ítemes no significaría un aumento de la estabilidad de la medición.

Tabla 5: Estadísticos de fiabilidad de la escala de Percepción de Atributos de la Sociedad Chilena.

\begin{tabular}{|r|r|}
\hline $\begin{array}{c}\text { Alfa de } \\
\text { Cronbach }\end{array}$ & $\begin{array}{c}\text { N de } \\
\text { elementos }\end{array}$ \\
\hline, 881 & 10 \\
\hline
\end{tabular}

Tabla 6: Media, desviación típica, correlación ítem-total y a de Cronbach sin el ítem para cada ítem de la escala de Percepción de Atributos de la Sociedad Chilena.

\begin{tabular}{|c|c|c|c|c|}
\hline Ítem & Media & $\begin{array}{l}\text { Desviación } \\
\text { típica }\end{array}$ & $\begin{array}{c}\text { Correlación } \\
\text { ítem-total }\end{array}$ & $\begin{array}{c}\text { a de } \\
\text { Cronbach } \\
\text { sin el ítem }\end{array}$ \\
\hline Democrático & 4,460 & 2,325 & 0,537 & 0,874 \\
\hline Austero & 6,627 & 2,402 & 0,498 & 0,877 \\
\hline $\begin{array}{l}\text { Igualitario en } \\
\text { oportunidades }\end{array}$ & 6,596 & 2,790 & 0,650 & 0,866 \\
\hline Libre & 5,103 & 2,545 & 0,677 & 0,864 \\
\hline Tolerante & 6,336 & 2,596 & 0,731 & 0,860 \\
\hline No Clasista & 5,832 & 3,294 & 0,526 & 0,879 \\
\hline Solidario & 5,219 & 2,589 & 0,660 & 0,865 \\
\hline Justo & 6,018 & 2,779 & 0,649 & 0,866 \\
\hline Seguro & 5,365 & 2,333 & 0,591 & 0,871 \\
\hline Moderno & 5,380 & 2,310 & 0,611 & 0,870 \\
\hline
\end{tabular}




\section{Conclusiones}

En una evaluación general se sostiene que el presente artículo cumple con lo propuesto en la medida que se desarrolló un relato del proceso de validación del cuestionario utilizado en la ENCED y las complejidades enfrentadas durante este proceder. Es importante recalcar la variedad de instancias en las que el instrumento de medición se sometió a validación: revisión teórica, análisis de expertos, aplicación de prueba piloto, etc.

Por otra parte, se realizó una serie de análisis de fiabilidad de las escalas incluidas en el cuestionario. Las escalas a evaluar fueron cuatro: Escala de Estrategias de Adquisición de Información; Escala de Autoritarismo de Derechas; Escala de LiberalismoConservadurismo y Escala de Percepción de Atributos de la Sociedad Chilena.

Cabe mencionar los altos números registrados en los 4 análisis de consistencia interna desarrollados. El coeficiente alpha de Cronbach mostró números superiores a 0.77 en todas las escalas, por lo que se deduce que los ítems considerados en la construcción de las escalas aseguran estabilidad en la medición. De hecho, el análisis de consistencia interna en la escala de percepción de atributos de la sociedad chilena se alzó sobre 0.88 , siendo el número más alto registrado.

Esta situación cobra importancia en la medida que es una de las 2 escalas que no habían sido testeadas con anterioridad, por lo que supone una buena línea de base para que investigadores puedan seguir ahondando en la medición de estos atributos. La misma tendencia se repite para la escala de liberalismo-conservadurismo en tanto es una escala creada especialmente para los estudiantes de Derecho.

Finalmente, cabe reforzar que tanto la escala de estrategias de adquisición de información como la escala de autoritarismo de derechas mostraron una consistencia interna similar a los niveles consultados en las bibliografía que sirvió de base para la construcción del cuestionario.

Estos resultados que parecen únicamente necesarios para la prosecución de la encuesta, resultan interesantes en la medida que las pretensiones del presente estudio no sobrepasan el alcance exploratorio-descriptivo, por lo que se abre un nuevo campo de análisis en torno a la validación de instrumentos de medición cuantitativos dedicados exclusivamente al ámbito de la investigación jurídica. 


\section{Referencias Bibliográficas}

ALBERT GÓMEZ, María José. La Investigación Educativa: Claves Teóricas. Madrid: Mc Graw Hill, 2007.

ALTEMEYER, Bob. Right-Wing Authoritarianism. Winnipeg: University of Manitoba Press, 1981.

ASÚN INOSTROZA, Rodrigo. "Construcción de cuestionarios y escalas: el proceso de la producción de información cuantitativa". En: Metodologías de Investigación Social: Introducción a Los Oficios. Santiago: LOM Ediciones, 2014.

BEST, John W. Cómo Investigar en Educación. Madrid: Ediciones Morata, 1992.

CÁRDENAS, Manuel y PARRA, Luis; “Adaptación y validación de la versión abreviada de la escala de autoritarismos de derechas (RWA) en una muestra chilena”. En: Revista de Psicología, Universidad de Chile. 2010, vol. 19, núm. 1.

CENTRO DE ESTUDIOS DE LA FEDERACIÓN DE ESTUDIANTES DE LA UNIVERSIDAD DE CHILE. Encuesta de Caracterización de Estudiantes Universidad de Chile 2011: Análisis y Principales Resultados, 2012. Disponible ne web: http://fech.cl/wpcontent/uploads/2012/03/INFORME-FINAL-ENCUESTA-CEFECH.pdf [consultado en diciembre de 2014]

CLOSAS, Antonio Humberto et al. "Validación de un cuestionario sobre estrategias de aprendizaje para estudiantes universitarios". En: SOSA, Carola y DALFARO, Nidia (comps.). La Universidad Tecnológica Nacional - U.T.N. - En El NorEste Argentino - N.E.A. Disponible en web: $\quad$ http://www.edutecne.utn.edu.ar/investigacion_fr_res/Closas_Ciencias_Sociales.pdf [consultado en diciembre de 2014]

CORBETTA, Piergiorgio. Metodología y técnicas de investigación social. Madrid: Mc Graw Hill, 2003.

COZBY, Paul C. Métodos de Investigación del Comportamiento. México: Ed. McGraw Hill, 2004.

HERNÁNDEZ SAMPIERI, Roberto; FERNÁNDEZ COLLADO, Carlos y BAPTISTA, Lucio Pilar. Metodología de la Investigación. México: Ed. McGraw Hill, 2006.

INJUV, GOBIERNO DE CHILE; Sexta Encuesta Nacional de Juventud. Disponible en web http://www.injuv.gob.cl/modules.php [consultado en diciembre de 2014]

PARDINAS, Felipe. Metodología y técnicas de investigación en ciencias sociales. México: Siglo Veintiuno Editores, 2008.

ROMÁN, José María y GALLEGO, Sagrario. Escala de estrategias de aprendizaje. Madrid: TEA Ediciones, 1994.

SIERRA, Restituto. Técnicas de investigación social: teoría y ejercicios. Madrid: Thomson Editores, 2007. 